\title{
The curvature of cucumber fruits is associated with spatial variation in auxin accumulation and expression of a YUCCA biosynthesis gene
}

\author{
Shengnan Li ${ }^{1}$, Chunhua Wang ${ }^{1,2}$, Xiuyan Zhou' ${ }^{1}$ Dong Liu', Chunhong Liu', Jie Luan', Zhiwei Qin ${ }^{1}$ and Ming Xin
}

\begin{abstract}
Fruit curving lowers the commercial value of cucumber and leads to significant economic losses. The mechanism driving the abnormal curving of cucumber is largely unknown. Through our previous work, we discovered that 2 days post-anthesis (DPA) was the key time point at which various phenotypic and genotypic characteristics of cucumber fruits are determined. Here, we analyzed the transcriptome of the concave (C1) and convex (C2) sides of curved fruits at 2 DPA by Gene Ontology (GO) enrichment and functional pathway enrichment analyses and identified auxin as a putative factor influencing fruit curvature. Changes in the curve angle in the fruits and exogenous auxin treatment analyses showed that asymmetric auxin distribution induces fruit curving. Identification of differentially expressed genes (DEGs) related to auxin and GPCR validation showed that CSYUC10b had the most significant differential expression when both sides of the curved fruits were compared. Gene functional analysis showed that the transcript levels of CSYUC10b and the auxin concentration were even on both sides of the fruit in CsYUC10b-overexpressing plants, which in turn contributed to an equal rate of growth of both sides of cucumber fruits and resulted in a straight shape of the fruits. Thus, we conclude that CsYUC10b promotes the formation of straight cucumber fruits, with possible applications in the production and breeding of cucumber.
\end{abstract}

\section{Introduction}

Fruit shape is a critical factor determining commercial production, germplasm accession classification, and consumer preference. Cucumber (Cucumis sativus L.) is an economically important vegetable species of the family Cucurbitaceae and produces both straight and curved fruits. However, fruit curving severely reduces its commercial and economic value ${ }^{1}$. Cucumber fruit shape is controlled by multiple genes and therefore exhibits quantitative inheritance ${ }^{2}$. In addition, different genotypic varieties of cucumber differ significantly in terms of the proportion of curved fruits versus straight fruits ${ }^{3}$.

\footnotetext{
Correspondence: Ming Xin (xinmingneau@126.com)

${ }^{1}$ College of Horticulture and Landscape Architecture, Key Laboratory of Biology and Genetic Improvement of Horticultural Crops (Northeast Region), Northeast Agricultural University, Harbin 150030, China

${ }^{2}$ College of Horticulture, Fujian Provincial Key Laboratory of Haixia Applied

Plant Systems Biology, Fujian Agriculture and Forestry University, Fuzhou 350002, China

These authors contributed equally: Shengnan Li, Chunhua Wang
}

However, little is known about the mechanisms underlying fruit curving, which is a crucial factor for developing new varieties.

The growth of cucumber fruit can be separated into cell division, fruit elongation, expansion, and ripening stages ${ }^{4}$. The fruit shape obtained at the end of this process depends on the ovary shape and is controlled by preanthesis processes ${ }^{5}$. In addition, several hormones regulate fruit elongation and shape in response to environmental stimuli ${ }^{6}$. For instance, the endogenous level of auxin is closely associated with fruit shape, set, and size ${ }^{7}$. Downregulation of auxin response factor 7 (SlARF7) in tomato (Solanum lycopersicum) results in heart-shaped fruits with a thick pericarp ${ }^{8}$. Auxin homeostasis depends on the balance between its biosynthesis, metabolism, and inter- and intracellular transport ${ }^{9}$. In Arabidopsis thaliana and maize (Zea mays), auxin biosynthesis involves a two-step pathway starting with the conversion of tryptophan to indole-3-pyruvate (IPA) by

\section{(c) The Author(s) 2020}

(c) (i) Open Access This article is licensed under a Creative Commons Attribution 4.0 International License, which permits use, sharing, adaptation, distribution and reproduction cc) in any medium or format, as long as you give appropriate credit to the original author(s) and the source, provide a link to the Creative Commons license, and indicate if changes were made. The images or other third party material in this article are included in the article's Creative Commons license, unless indicated otherwise in a credit line to the material. If material is not included in the article's Creative Commons license and your intended use is not permitted by statutory regulation or exceeds the permitted use, you will need to obtain permission directly from the copyright holder. To view a copy of this license, visit http://creativecommons.org/licenses/by/4.0/. 
tryptophan aminotransferase (TAR2), a member of the aminotransferase Arabidopsis (TAA) family, followed by catalysis of IPA into the major endogenous auxin indole3 -acetic acid (IAA) by YUCCA flavin-containing monooxygenases $^{10}$. In addition, weak insensitive 2 (WEI2) and weak ethylene insensitive 7 (WEI7) are known ratelimiting enzymes involved in the biosynthesis of the auxin precursor tryptophan. Loss of WEI2 and WEI7 has no obvious effect on auxin phenotypes; however, upregulation of WEI2 and WEI7 contributes to auxin accumulation in the roots ${ }^{11}$. The spatiotemporal distribution of auxin is controlled by auxin resistant 1 (AUX1), like aux (LAX1-3) proteins mediate auxin influx, and PIN-formed (PIN1-8) mediates auxin efflux, all of which collectively regulate organ-level auxin transport ${ }^{12,13}$.

Auxin plays an important role in apical hook development in A. thaliana by inducing differential cell growth on both sides of the hook. Higher auxin concentration on the inner side of the hook compared with the outer side correlates to reduced cell elongation ${ }^{14}$. In addition, the auxin biosynthesis genes YUCCA1, TAA1/WEI8, and $T A R 2$ are upregulated in the hook region, and the wei8, tar2, and yuc1/2/4/6 mutants of A. thaliana display improper apical hook formation ${ }^{15,16}$. Furthermore, the auxin transporter genes AUX1 and LAX3 and auxin response factors (ARFs) also play an essential role in differential hook growth ${ }^{17}$. Ethylene enhances auxin biosynthesis in the inner side of the hook by upregulating TAR2, IAA3, IAA12, and IAA13, resulting in differential cell growth. This hormone also stimulates hypocotyl elongation upon light exposure by activating IAA and upregulating TAA1, YUCCA1, and YUCCA5 as well as the auxin transport-related genes $A U X 1, P G P 19, P I N 3$, and $P G P 1^{18}$. In a previous study, we analyzed the transcriptomes of curved cucumber fruits retrieved from the expressed sequence tag database ${ }^{19}$ and identified a putative auxin synthesis gene, CsYUC10b (Csa3G190380), that was likely associated with fruit curvature. Herein, we found that CsYUC1Ob induced the formation of straight fruit by promoting consistent bilateral auxin biosynthesis in the fruits.

\section{Materials and methods Plants}

Cucumber varieties L18, D0328-3, and D0859, with different genotypes and respective fruit curving ratios of $52.56,39.28$, and 28.21 , were selected for the study ${ }^{20}$. The seeds of late-generation inbred lines from our laboratory were sown in pots containing a 1:1 mixture of soil and substrate. For the L18, D0328-3, and D0859 genotypes, 150,50 , and 50 plants were grown, respectively, in a controlled chamber at Northeast Agricultural University under a 12 -h photoperiod at $29^{\circ} \mathrm{C} / 17^{\circ} \mathrm{C}$ (day/night) and $75 \%$ relative humidity. The flowers were manually pollinated. L18 produced 3-4 fruits per plant, and a total of 580 fruits, including 308 curved fruits and 272 straight fruits, were used in this study. The number of fruits produced by the D0328-3 and D0859 genotypes was 220 (of which 86 were curved) and 200 (of which 56 were curved), respectively. The detailed data are shown in Supplementary Table S1. The nodes of 8-15 fruits from five individual plants showing consistent growth at 2, 4, 6, 8 , and 10 days post-anthesis (DPA) were collected, flashfrozen in liquid nitrogen, and stored at $-80^{\circ} \mathrm{C}$. The $\mathrm{L} 18$ $\mathrm{T}_{2}$ generation inbred lines OX4, OX7, and OX10 were used for transgenic experiments, and 50 plants of each line were grown under similar conditions.

\section{Library construction and transcriptome analysis}

A study by Wang et al. ${ }^{21}$ showed that 2 DPA was a key time point to determine whether cucumber fruits would become curved or straight. A transcriptome expression profile analysis of curved (including the concave and convex sides) and straight fruits at 2 DPA showed that upregulated genes on the convex side were important determinants of cucumber fruit curvature ${ }^{21}$. Consequently, in this study, we focused on the difference between the concave $(\mathrm{C} 1)$ and convex $(\mathrm{C} 2)$ sides of the curved fruits at $2 \mathrm{DPA}$. The exocarps $(0.1 \mathrm{~cm}$ thick) were dissected from the $\mathrm{C} 1$ and $\mathrm{C} 2$ sides of nine curved fruits of the L18 variety at 2 DPA. These exocarps were used for RNA extraction using TRIzol reagent (Invitrogen, Carlsbad, CA, USA). The quality and quantity of the purified RNA were then determined by measuring the absorbance at $260 \mathrm{~nm} / 280 \mathrm{~nm}$ (A260/A280) using a SmartSpec Plus instrument (BioRad, USA). The RNA integrity was further verified by $1.5 \%$ agarose gel electrophoresis. For each sample, $3 \mathrm{~g}$ of total RNA was used for RNA-seq library preparation. The libraries were prepared according to the manufacturer's instructions and subjected to an Illumina GAIIx system for 80 nt single-end sequencing by ABlife, Inc. (Wuhan, China) or to a HiSeq 2000 system for $100 \mathrm{nt}$ pair-end sequencing by BGI, Inc. (Shenzhen, China).

Information in the Sequence Read Archive database (accession number: SRP111902) was used for transcriptome analysis ${ }^{21}$. After trimming the adaptor sequences, we obtained clean raw reads from the raw sequence reads, which were filtered based on base quality $(Q \geq 20)^{22}$. All the clean reads were aligned to the reference genome (http://cucurbitgenomics.org/) of cucumber (Chinese Long genome V2) by TopHat2 (http://tophat. cbcb.umd.edu/). All the values were expressed in reads per kilobase pair per million reads (RPKM) based on the read and gene locations in the genome. Differentially expressed genes (DEGs) were chosen based on the following criteria: $p$ value $<0.01, \mid \log 2$ ratio $\geq 1 \mid$ and false discovery rate $(\mathrm{FDR})<0.05$. Gene Ontology (GO) enrichment analysis of the DEGs was implemented by the 
agriGO package (http://bioinfo.cau.edu.cn/agriGO/index. php). An enrichment score (ES) of $\geq 1.3$ and $p<0.05$ was considered significant ${ }^{23}$. Gene set enrichment analysis (GSEA) was used to analyze gene sets and biological pathways by KOBAS $3.0^{24}$.

\section{Real-time quantitative PCR}

The exocarps of both sides of L18 curved fruits at $0-10$ DPA were used to verify the transcriptomic results, and samples of roots, stems, leaves, stem apices, female flowers (blooming), and male flowers (blooming) were also measured. Fruits were sampled from L18 to analyze the expression of CsYUC1Ob. Total RNA was isolated using TRIzol reagent (Invitrogen) according to the manufacturer's instructions and reverse transcribed to cDNA using a kit (Toyobo, Japan). qRT-PCR was performed using $10 \mu \mathrm{L}$ of $2 \mathrm{x}$ Fast qPCR Master Mixture (DiNing, China), $0.5 \mu \mathrm{L}$ each of the forward and reverse primers $(10 \mu \mathrm{M}), 2 \mu \mathrm{L}$ of cDNA template and $\mathrm{ddH}_{2} \mathrm{O}$ such that the total volume was $20 \mu \mathrm{L}$. The reaction program was as follows: initial denaturation at $95^{\circ} \mathrm{C}$ for $3 \mathrm{~min}$; by 40 cycles of denaturation at $95^{\circ} \mathrm{C}$ for $10 \mathrm{~s}$, annealing at $56^{\circ} \mathrm{C}$ for $30 \mathrm{~s}$ and elongation at $72{ }^{\circ} \mathrm{C}$ for $30 \mathrm{~s}$; and a final extension at $72^{\circ} \mathrm{C}$. The fluorescence signals were analyzed using qTOWER 2.0 (AnalytikJena, Germany). The relative expression of the candidate genes relative to the internal control $E F 1 a^{25}$ was calculated using the $2^{-\Delta \Delta C T}$ method $^{26}$. Three biological and technical replicates were analyzed. The primers used are listed in Supplementary Table S2.

\section{Subcellular localization}

The coding sequences of CsYUC1Ob excluding termination codons (TAG) were amplified by PCR, cleaved with HindIII and BamHI, and then cloned into a pGIIEGFP expression plasmid using T4 DNA ligase (Invitrogen $)^{27}$. The recombinant 35S:CsYUC10b-GFP plasmid and empty plasmids were transformed into isolated protoplasts from $A$. thaliana ${ }^{28}$, which were then observed under a confocal spectral microscope equipped with 488 and $580 \mathrm{~nm}$ filters (Leica, Germany).

\section{Generation of CsYUC10b transgenic cucumber}

The amplified CsYUC1Ob sequence was cloned into a p1250 vector harboring a glyphosate resistance gene ${ }^{29}$ under a constitutive promoter following $\mathrm{XcmI}$ digestion and $\mathrm{T}_{4}$ ligation (Invitrogen). The p1250-CsYUC1Ob and empty plasmids were transformed into Agrobacterium LBA4404, which was then used to infect the cotyledons of L18 plants ${ }^{30}$. The cloned cotyledons were regenerated as described previously ${ }^{31}$, and the transgenic lines were screened in MS media supplemented with $1 \mathrm{mg} / \mathrm{L}$ glufosinate $^{32} \cdot T_{1}$ and $T_{2}$ plants were identified by PCR and qRT-PCR using specific primers (Supplementary Table
S2). Samples were collected from five individual plants for each biological repeat, and three biological and technical repeats were tested. The change in the curve angle was observed from 0 to 12 DPA between the CsYUC10b overexpression fruits and the control group fruits. The exocarps were collected from both sides of the fruits at 4 DPA to analyze the auxin content and the CsYUC1Ob expression levels.

\section{Treatment with 1-naphthaleneacetic acid (NAA), 1- naphthoxyacetic acid (NOA) and aminoethoxyvinylglycine (AVG)}

The ovaries of the straight and curved L18 fruits were sprayed at preanthesis (0 DPA) on one side (the concave side for the curved fruit) with $0.1,0.15$, and $0.20 \mu \mathrm{M}$ NAA (a synthetic auxin); $0.1 \mu \mathrm{M}$ AVG (an auxin biosynthesis inhibitor); or $0.1 \mu \mathrm{M}$ NOA (an auxin transport inhibitor). At the same time, both sides of the curved fruits were sprayed with the abovementioned solutions. The controls were sprayed with the same volume of water $(10 \%$ Tween was used as an adsorbent). The NAA stock was $5 \mathrm{mmol} / \mathrm{L}$, and the working volumes of NAA used were 20,30 , and $40 \mu \mathrm{L}$, with $10 \%$ Tween. Similarly, the volume of NOA and AVG was $20 \mu \mathrm{L}$, with $10 \%$ Tween. We sprayed the solutions using an even-bore squirt bottle, and each fruit was treated only once. The exocarps were collected from both sides of the suitably treated fruits at 4 DPA to analyze the auxin content and transcript levels of CsYUC10b. The change in the curve angle was observed from 0 to 12 DPA.

\section{Determination of auxin content}

The exocarp was collected from both sides of straight and curved fruits of the transgenic and control L18 plants at $0,2,4,6,8$, and 10 DPA. Auxin was extracted according to the protocol of Weiler ${ }^{33}$ and measured using an ELISA kit specific for IAA (Meimian Industrial Co., Ltd, China). Six replicates were tested per sample, and the experiment was performed three times. The IAA levels in the different groups were compared by Student's $t$-tests $(p<0.05)$.

\section{Results \\ Transcriptome analysis of curved cucumber fruits}

Transcriptome analysis revealed 27.07 and 20.59 million raw reads from $\mathrm{C} 1$ (clean reads, $85.71 \%$ ) and $\mathrm{C} 2$ (clean reads, $85.71 \%$ ) after low-quality adaptor and barcode sequences were removed (Supplementary Table S3). The sequencing quality analysis, sequence content distribution, and length distribution of the clean reads indicated good sequencing quality by RNA-seq (Supplementary Fig. S1). A total of 4313 DEGs were identified between the C1 (convex) and C2 (concave) sides of the curved fruits, of which 2351 were upregulated and 1962 were downregulated in $\mathrm{C} 1$ relative to $\mathrm{C} 2$ (Supplementary Fig. S2). Using ES $\geq 1.3$ and $p$ value $<0.05$ as criteria, 15 categories 
of significantly enriched genes were identified by GO analysis in $\mathrm{C} 2$, including 9 related to photosynthesis, adaxial/abaxial formation, redox processes and responses to auxin stimuli (Supplementary Table S4), suggesting the possible involvement of light in fruit curving. Likewise, the $\mathrm{C} 1$-specific categories were enriched in protein kinase activity, the ethylene-mediated signal pathway, regulation of cell size, regulation of cell shape, and polar auxin transport (PAT). In addition, several of the identified DEGs were related to plant hormones, including ethylene, auxin, cytokinin, abscisic acid, and gibberellins. The ethylene signal transduction-related genes were expressed predominantly in the fruits, followed by the genes coding for auxin production (Supplementary Table S5). In a previous report, ethylene was found to promote cucumber fruit curving ${ }^{21}$. Further functional pathway enrichment analysis showed that tryptophan metabolism, ribosomes, phenylpropanoid biosynthesis, and six other pathways had significant enrichment (Fig. 1). Importantly, tryptophan was the major auxin biosynthetic precursor, as inferred through our experiments. Thus, we surmised that auxin is also involved in fruit curving.

\section{Asymmetric auxin levels are correlated with cucumber fruit curving}

The curve angle of the L18 fruits differed between $0-20^{\circ}, 20-60^{\circ}$, and $\geq 60^{\circ}$ during development from 0 to 18 DPA. The increase was gradual between 0-4 DPA and peaked at 4-7 DPA, after which the angle decreased slowly from 8 to 18 DPA (Supplementary Fig. S3a). Accordingly, we divided the fruit curving process into the formation, maintenance, and opening phases. A curve angle of $0-20^{\circ}$ at 0 DPA correlated to straight fruit, while an angle $>20^{\circ}$ was indicative of a curved shape (Supplementary Fig. S3b), indicating that ovary growth plays a decisive factor in early curving. To determine the role of auxin in the development of curved fruits, we measured auxin levels on both sides of curved and straight fruits from 2 to 8 DPA. Auxin levels were markedly higher on the convex side than on the concave side of the curved fruits, peaking at 4 DPA and gradually declining thereafter. In contrast, both sides of the straight fruits had similar auxin levels throughout development (0-10 DPA) (Fig. 2a). The auxin concentration was 2.01-, 1.54- and 1.37-fold higher on the convex side than on the concave side in the fruits of the L18, D0859, and D0328-3 varieties, respectively, at 4 DPA; these results correlate with the respective curved fruit ratios of $52.56 \%, 39.28 \%$, and $28.21 \%$ in these genotypic varieties. Not surprisingly, both sides of the straight fruits showed similar auxin concentrations regardless of genotype (Fig. 2b). Overall, asymmetric auxin distribution likely induces cucumber fruit curving through differential growth of the two sides.

\section{Auxin distribution directly controls fruit curving}

Asymmetric auxin distribution during fruit curving is likely due to its aberrant local synthesis and polar transport. To test this hypothesis, we treated one side of the ovaries from straight fruits with synthetic auxin (NAA) at 0 DPA and observed faster growth of the fruit on the treated versus the untreated side, eventually resulting in a curved shape. The maximum curvature (approximately $155^{\circ}$ ) was observed in fruits treated with $0.2 \mu \mathrm{M}$ NAA at 6 DPA (Fig. 3a). Interestingly, the application of $1 \mu \mathrm{M}$ NAA

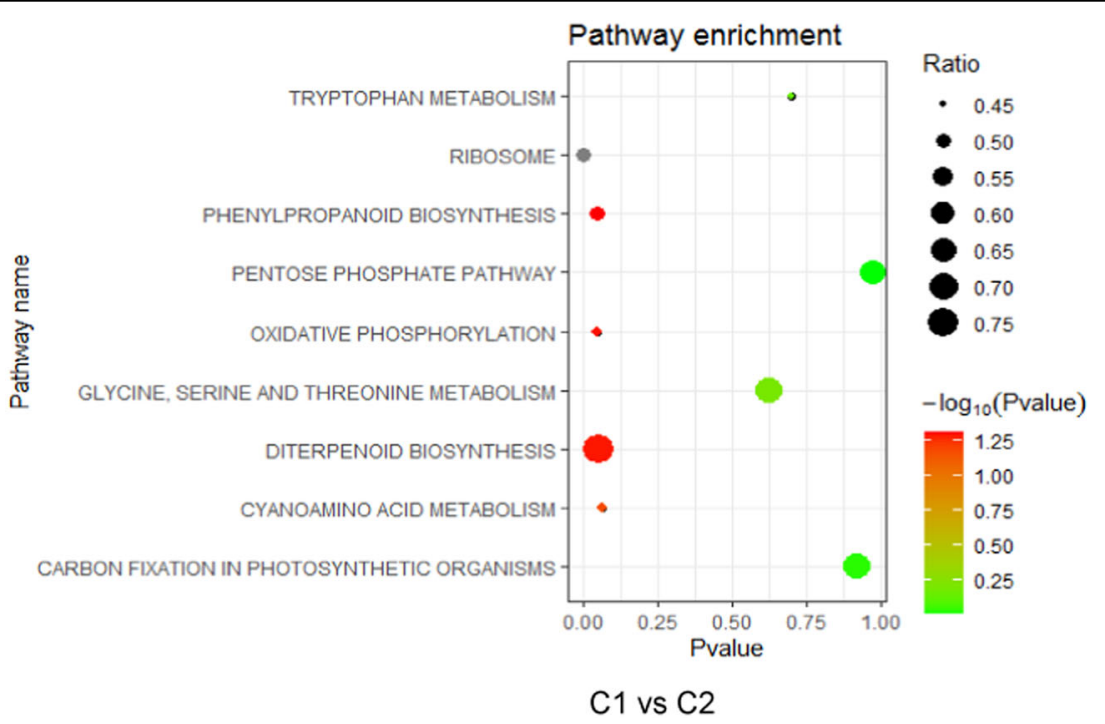

Fig. 1 Gene Ontology (GO) enrichement analysis of specific DEGs. Functional pathway enrichment on the concave (C1) and convex (C2) sides of curved fruits at 2 DPA according to GSEA 

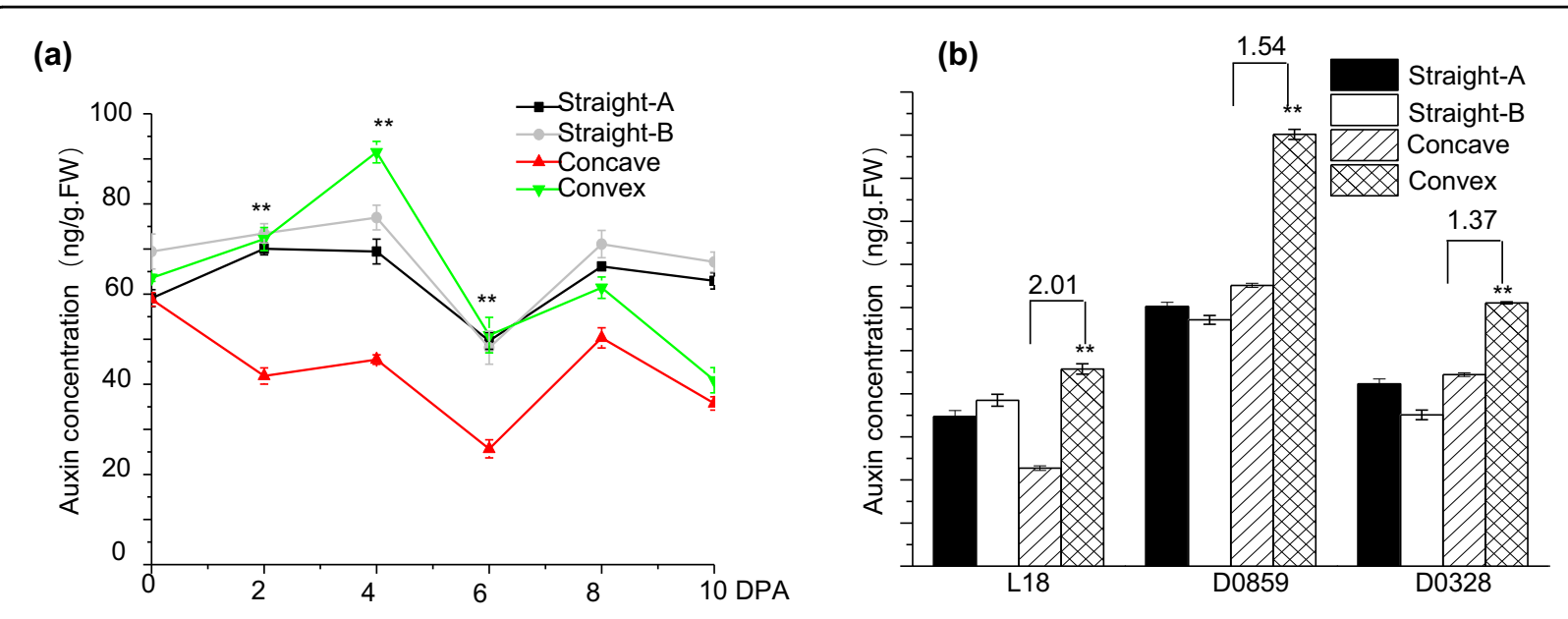

Fig. 2 Temporal changes in auxin concentration in cucumber fruits. a Auxin concentration on the $\mathrm{C} 1$ and $\mathrm{C} 2$ sides of curved fruits and on both sides of straight fruits at 2, 4, 6, 8, and 10 DPA. $\mathbf{b}$ Auxin concentration on the $\mathrm{C} 1$ and $\mathrm{C} 2$ sides of curved fruits and on both sides of straight fruits of the L18 (52.56\%), D0859 (39.28\%) and D0328-3 (28.21\%) genotypes at 4 DPA. The data are the means ( \pm SEs) of three independent experiments, with five replicates each $(p<0.05)$

(a)

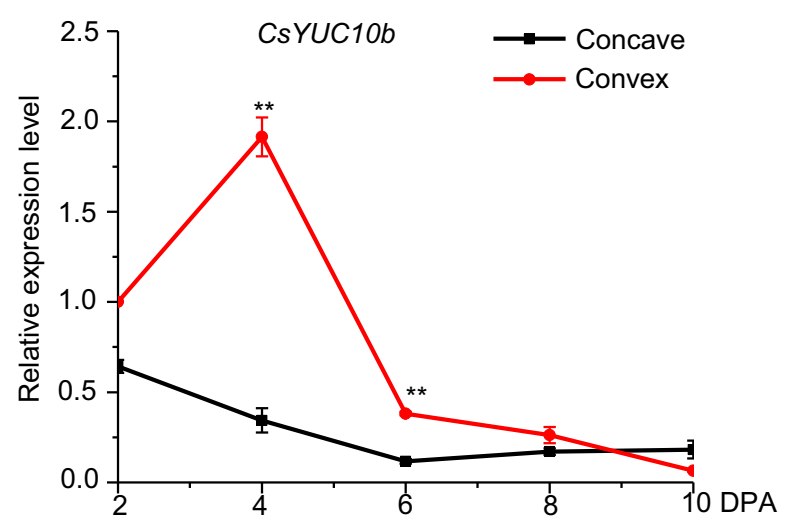

(c)

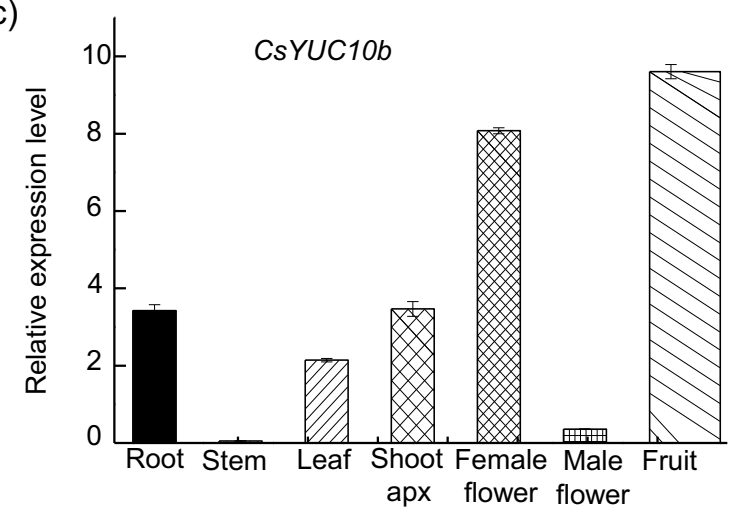

(b)

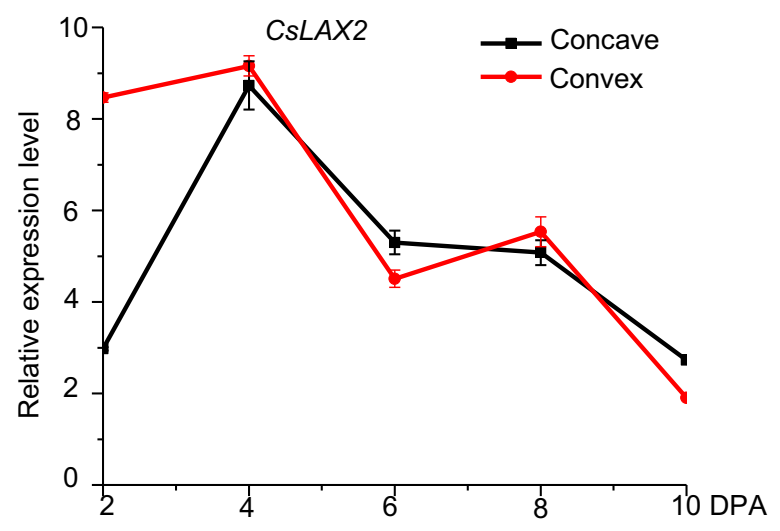

(d)

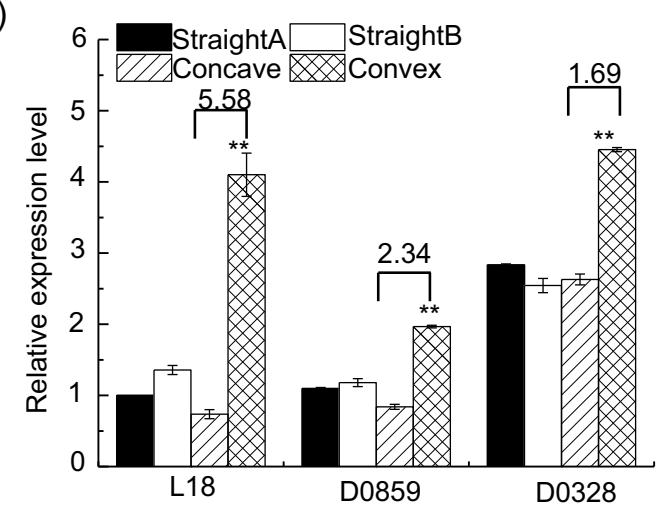

Fig. 3 Expression patterns of auxin-related genes in cucumber. a CSYUC10b mRNA levels on the concave and convex sides at 2, 4, 6, 8 and 10 DPA. b CSLAX2 mRNA levels on the concave and convex sides at 2, 4, 6, 8, and 10 DPA. c CsYUC10b levels in different tissues of L18 plants. d CsYUC10b levels in curved and straight fruits of the L18, D0859 and D0328-3 genotypes at 4 DPA. CSEF1a was used as a reference gene. The data are the means ( \pm SEs) of three independent experiments, with five replicates each $(P<0.05) .{ }^{*} P<0.05$, and $\left.{ }^{* *} P<0.01\right)$ 
on the concave side of ovaries with a $45^{\circ}$ curve decreased the curvature at $4 \mathrm{DPA}$, and the fruits ultimately had a straight shape (Fig. 3b). Furthermore, when ovaries with $70-90^{\circ}$ curving were treated with $0.1,0.15$, and $0.2 \mu \mathrm{M}$ NAA on the concave side, the higher doses were able to decrease the angle of curvature at 2 DPA; however, while $0.15 \mu \mathrm{M}$ NAA restored the straight shape of the final fruit, $0.2 \mu \mathrm{M}$ NAA resulted in a backward curvature (Fig. 3c). Through further analysis, we found that in response to the application of $0.15 \mu \mathrm{M}$ NAA, the auxin content was consistent on both sides of fruits at 4 DPA (Fig. 3d). Consistent with these findings, the application of the auxin transport inhibitor NOA to both sides of curved ovaries decreased the angle of curvature (Fig. 3e), as well as the differential bilateral auxin levels (Fig. 2f), compared to that of the untreated control at 4 DPA. To further support our conclusions, we examined the impact of the auxin biosynthesis inhibitor AVG). Following the application of AVG $(0.1 \mu \mathrm{M})$ to the concave side of the ovary during the preanthesis stage, we observed that the fruit exhibited a relatively straight phenotype at 4 DPA (Supplementary Fig. S4). This approach did not achieve the desired effect because both ethylene and auxin synthesis were inhibited by AVG, and ethylene has been reported to contribute to cucumber fruit curving ${ }^{21}$. Overall, asymmetric auxin distribution led to uneven growth of both sides of the fruit, resulting in the formation of curved fruits.

\section{CsYUC10b plays a crucial role in fruit curving}

We identified 33 auxin homeostasis-related genes from the transcriptome data of curved fruit exocarps (Table 1), of which YUC10b (Csa3G190380), GH3.6 (Csa3G431430), AUX1 (Csa3G731880) and LAX2 (Csa2G264590) were differentially expressed between $\mathrm{C} 1$ and $\mathrm{C} 2(\mid \log 2$ ratio $\geq$ $1 \mid$ and $p$ value $=0.01$ ), and only CsYUC1Ob and CsLAX2 were upregulated on the convex side compared to the concave side of the fruits. The transcript levels were measured in the curved fruits from 0 to $10 \mathrm{DPA}$, and we observed a marked upregulation of CsYUC1Ob on the convex side compared with the concave side at 2-8 DPA, which was consistent with the maximum curvature recorded at 4 DPA (Fig. 4a). However, CsLAX2 expression was similar on both sides of the curved fruits from 4 to 10 DPA (Fig. 4b). Thus, we hypothesized that CsYUC10b is a key gene involved in cucumber fruit curving. We also analyzed the expression of $C s Y U C 10 b$ in all the organs of the L18 plants and detected low levels of transcripts in the male flower buds and stems; moderate levels in the roots, leaves and shoot apices; and increased levels in open female flowers. The highest levels were detected in the fruits (Fig. 4c). Furthermore, the CsYUC1Ob transcript levels were 5.58-, 2.34- and 1.69-fold higher in the convex side compared with the concave side of curved fruits from the L18, D0859, and D0328-3 plants, respectively, at 4 DPA, while no significant differences were detected on either side of the straight fruits (Fig. 4d).

\section{CsYUC10b cloning and expression}

To further investigate the role of CsYUC1Ob in fruit curving, we analyzed its full-length (1158 bp) CDS (Csa3M190380.1), which encodes a putative 385 amino acid protein. As shown in Supplementary Fig. S5a, CsYUC10b has a 171 bp $5^{\prime}$ untranslated region (UTR), a $188 \mathrm{bp} \mathrm{3 \prime}$ UTR, four exons and three introns. Phylogenetic tree analysis (Supplementary Fig. S5b) showed that $C s Y U C 10 b$ is orthologous to the YUC genes of Cucumis melo (CmYUC10), Morus notabilis (MnYUC10), Theobroma cacao (TcYUC10) and Glycine soja (GsYUC10), with respective sequence homologies of $90.39 \%, 64.25 \%$, $61.66 \%$ and $58.29 \%$ (Supplementary Fig. S5c). The $C s Y U C 10 b$ sequence was cloned into an expression vector under the control of the constitutive $35 \mathrm{~S}$ promoter and expressed in A. thaliana protoplasts. As shown in Supplementary Fig. S6, the CsYUC10b-GFP fusion protein was localized in the cytoplasm, whereas the empty plasmid controls showed diffuse fluorescence.

\section{CsYUC10b promotes straight fruit development in cucumber by balancing auxin biosynthesis}

Our findings thus far indicated that asymmetric auxin accumulation in cucumber fruits caused uneven growth and eventual curving. To test this hypothesis, we generated CsYUC10b-overexpressing transgenic cucumber lines, screened the resistant plants with $1 \mathrm{mg} / \mathrm{L}$ glufosinate, and ultimately obtained forty-four resistant transgenic plants. Our PCR confirmation results showed that the whole identified $1800 \mathrm{bp}$ fragment included CsYUC10b, which was $1158 \mathrm{bp}$, and a $600 \mathrm{bp}$ fragment from the p1250 vector (Supplementary Fig. S7). Twelve PCR-positive plants that displayed increased growth were subjected to a qPCR-based analysis, the results of which showed that OX4, OX7, and OX10 expressed the highest levels; these plants were self-pollinated for subsequent studies in the $\mathrm{T}_{2}$ generation (Fig. 5a). The transgenic plants displayed phenotypes associated with auxin hyperaccumulation, such as downward curled leaves and rapid stem elongation at the seedling stage. (Fig. 5b). Furthermore, compared with the wild-type controls, the CsYUC1Ob transgenic plants showed faster growth of the ovaries of female flowers (Fig. 5c). As shown in Fig. 4d, the respective ovary lengths in the OX4, OX7, OX10 transgenic plants and control plants were 3.31, 3.15, 3.42, and $2.12 \mathrm{~cm}$. These results strongly indicated that CsYUC10b promotes auxin hyperaccumulation and ovary growth. Interestingly, at $4 \mathrm{DPA}$, the CsYUC10b-overexpressing plants produced straight fruits compared with the highly curved $\left(80^{\circ}\right)$ fruits of the control plants 
Table 1 List of 33 auxin homeostasis-related genes on the C1 and C2 sides of curved cucumber fruits

\begin{tabular}{|c|c|c|c|c|c|c|}
\hline \multirow[b]{2}{*}{ Gene ID } & \multirow[b]{2}{*}{$\log _{2}$ (ratio) $(\mathrm{C} 1 / \mathrm{C} 2)$} & \multirow[b]{2}{*}{$p$ value } & \multicolumn{2}{|l|}{ RPKM } & \multirow[b]{2}{*}{ Functional annotation } & \multirow[b]{2}{*}{ Gene family } \\
\hline & & & Concave (C1) & Convex (C2) & & \\
\hline Csa3G133910 & 0 & 1 & 0 & 0 & YUC3 & Flavin monooxygenase (YUC) \\
\hline Csa2G379350 & -0.920955361 & 2.91733E-06 & 3.82 & 6.07 & YUC4 & \\
\hline Csa1G242600 & 1.715031143 & 0.453125 & 0.29 & 0.07 & YUC6 & \\
\hline Csa2G375750 & -2.092323779 & 0.109375 & 0.07 & 0.27 & YUC6 & \\
\hline Csa3G619930 & 0 & 1 & 0 & 0 & YUC8 & \\
\hline Csa6G087870 & 28.11200867 & 0.0625 & 0.35 & 0 & YUC8 & \\
\hline Csa6G087880 & -0.470835402 & 0.377085587 & 2.15 & 2.51 & YUC8 & \\
\hline Csa3G190380 & -1.342856524 & $2.1685 E-193$ & 58.36 & 125.04 & YUC10 & \\
\hline Csa7G390100 & -0.751286861 & 0.049800114 & 1.13 & 1.65 & YUC10 & \\
\hline Csa6G454350 & -0.852544726 & $5.75218 \mathrm{E}-12$ & 8.23 & 12.58 & ILR1 & IAA-leucine resistant 1-like (ILL) \\
\hline Csa3G778230 & 0.251630622 & 0.181175894 & 7.06 & 5.01 & ILR1 & \\
\hline Csa3G778240 & -0.264276462 & 0.191899854 & 5.12 & 5.2 & ILR1 & \\
\hline CsaUNG029820 & 0 & 1 & 0 & 0 & ILL2 & \\
\hline Csa1G065960 & 0.036804848 & 0.74914208 & 22.16 & 18.26 & ILR3 & \\
\hline Csa2G423590 & -0.886864786 & $5.311 \mathrm{E}-54$ & 57.62 & 90.05 & ILR3 & \\
\hline Csa3G415100 & -0.014845026 & 0.917664014 & 21.01 & 17.96 & ILR3 & \\
\hline Csa3G416140 & 0.407463355 & 8.67334E-07 & 43.24 & 27.58 & ILR3 & \\
\hline Csa6G384060 & 0.939525087 & $6.7467 \mathrm{E}-06$ & 5.06 & 2.24 & ILL6 & \\
\hline Csa3G088930 & 27.52704617 & 0.125 & 0.09 & 0 & $\mathrm{GH} 3.6$ & Gretchen Hagen 3 (GH3) \\
\hline Csa3G431430 & 1.054517609 & $1.64345 \mathrm{E}-07$ & 5.48 & 2.27 & $\mathrm{GH} 3.6$ & \\
\hline Csa6G125240 & 25.52704617 & 1 & 0.02 & 0 & $\mathrm{GH} 3.6$ & \\
\hline Csa4G415930 & 28.84897427 & 0.0078125 & 0.26 & 0 & UGT74D1 & UDP-glucosyl transferase (UGT) \\
\hline Csa6G418940 & 25.52704617 & 1 & 0.06 & 0 & UGT74D1 & \\
\hline Csa1G025070 & -0.818359813 & 1.46901E-39 & 26.98 & 40.19 & PIN1 & PIN-formed (PIN) \\
\hline Csa1G042820 & -0.258996759 & 0.000400637 & 22.22 & 22.48 & PIN1 & \\
\hline Csa4G430820 & -0.175739787 & 0.369164706 & 3.84 & 3.66 & PIN1 & \\
\hline Csa5G576590 & 0.540204405 & $1.30289 E-05$ & 9.5 & 5.52 & PIN3 & \\
\hline Csa2G074170 & 0 & 1 & 0 & 0 & PIN5 & \\
\hline Csa5G284520 & -0.182890989 & 0.171454885 & 6.11 & 5.88 & PIN7 & \\
\hline Csa3G827360 & -0.5695301 & $3.64434 \mathrm{E}-06$ & 9.95 & 12.5 & PIN8 & \\
\hline Csa3G731880 & 1.731150808 & 4.41503E-33 & 11.09 & 2.85 & $A \cup X 1$ & Auxin resistant 1 (AUX1) \\
\hline Csa2G264590 & -3.221606796 & $1.33316 E-20$ & 0.5 & 3.88 & LAX2 & \\
\hline Csa7G010800 & 0.692825349 & 4.71397E-08 & 14.23 & 7.48 & LAX2 & \\
\hline
\end{tabular}

(Fig. 6a). Furthermore, the angle of fruit curvature decreased significantly for the transgenic plants compared to the control plants at 0-10 DPA (Fig. 6b). We observed $188,182,189$, and 176 fruits produced by the control, OX4, OX7, and OX10 lines (Supplementary Table S6). As shown in Fig. 6c, the proportion of curved fruits was only
$21.5,20.6$, and $19.9 \%$ for the OX4, OX7, and OX10 transgenic lines compared to $52.6 \%$ for the control plants (Fig. 6c). In addition, the CsYUC1Ob transcript levels and auxin concentration were significantly higher in the transgenic plants than in the control plants and showed a positive correlation (Fig. 6d, e). Consistent with these 
(a)

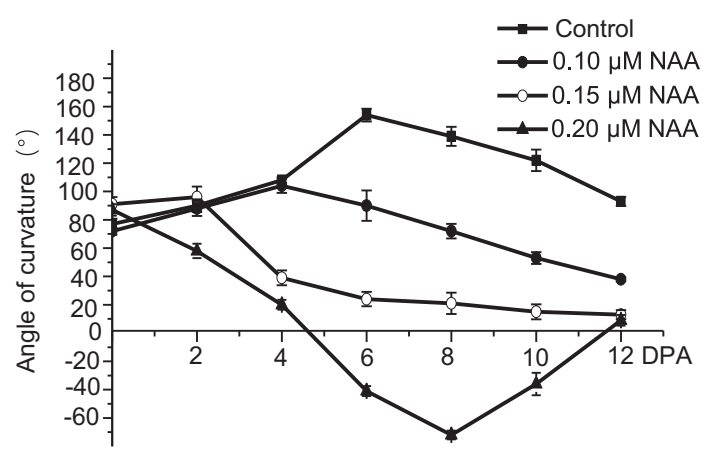

(c)

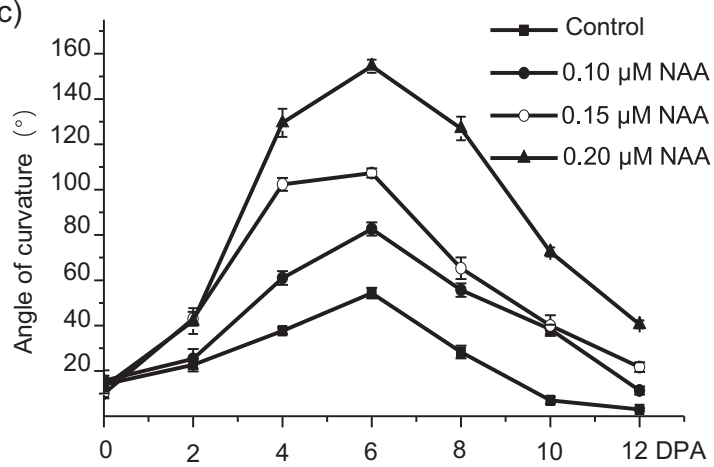

(e)

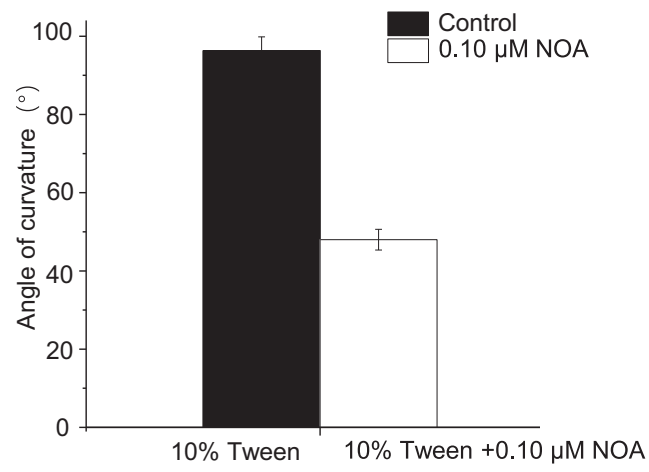

(b)

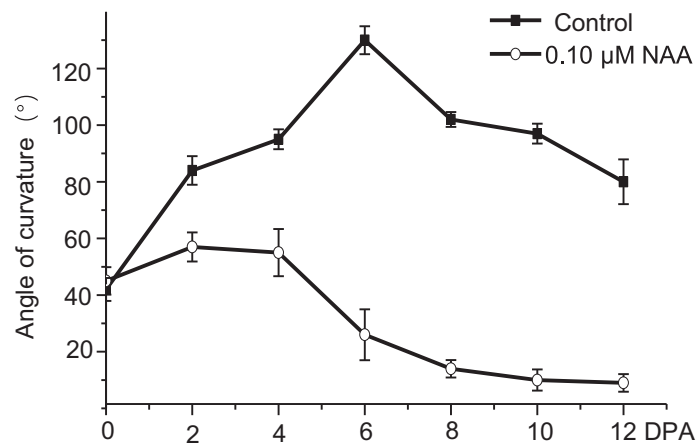

(d)

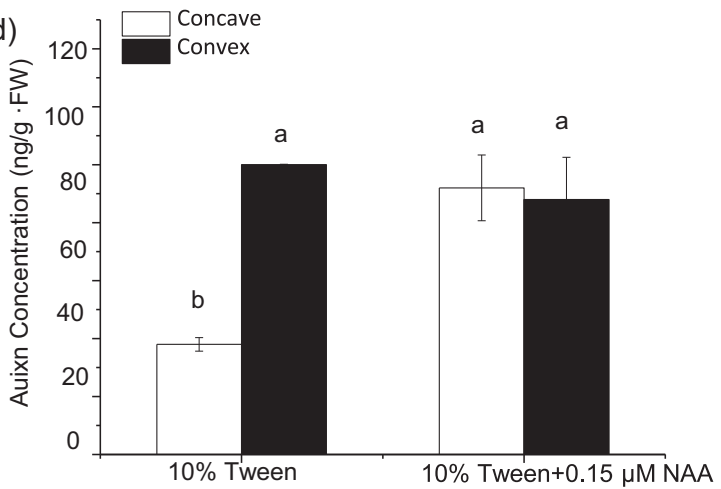

(f)

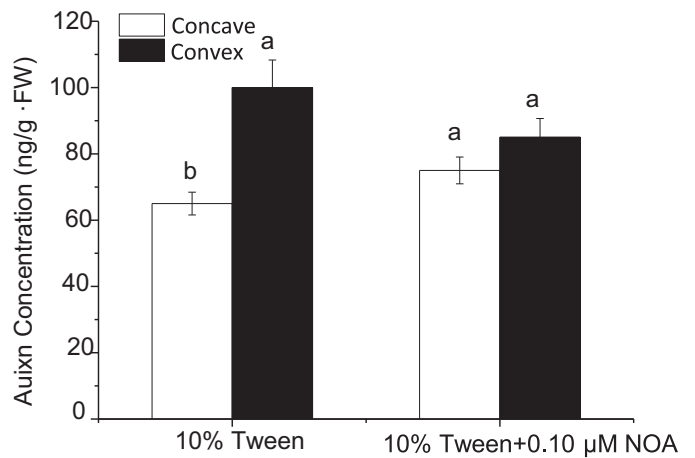

Fig. 4 Effects of exogenous auxin on cucumber fruits. a Angle of curvature of straight fruits treated with $0.1,0.15$, and $0.2 \mu M N A A$ at $2,4,6,8$, and 10 DPA. $\mathbf{b}$ Angle of curvature of $45^{\circ}$ curved fruits treated with $0.1 \mu \mathrm{M}$ NAA on the concave side at 2, 4, 6, 8, and 10 DPA. c Angle of curvature of 70-90 curved fruits treated with $0.1,0.15$, and $0.2 \mu \mathrm{M}$ NAA on the concave side at $2,4,6,8$, and 10 DPA. $\mathbf{d}$ Auxin concentration on the concave and convex sides of the control and $0.1 \mu \mathrm{M}$ NAA-treated fruits at 4 DPA. e Angle of curvature of curved fruits treated with $0.1 \mu \mathrm{M}$ NOA on both sides at 4 DPA. f Auxin concentration on the concave and convex sides of control and NOA-treated curved fruits at 4 DPA. CsEFla was used as a reference gene. The data are the means $( \pm$ SEs) of three independent experiments, with five replicates each $(p<0.05)$

findings, the auxin levels were even on both sides of the transgenic fruits, which resulted in a straight shape (Fig. 6a). Overall, CsYUC10b induces straight fruit development in cucumber by equilibrating auxin biosynthesis on both sides of the fruit.

\section{Discussion}

Asymmetric auxin distribution is a key factor driving the differential growth of plant organs. For instance, auxin efflux controls apical hook development in A. thaliana ${ }^{34}$, and significant auxin accumulation in the inner side of apical hook inhibits bean growth ${ }^{35}$. Furthermore, the application of exogenous auxin or the auxin transport inhibitor 1-naphthylcarbamylbenzoic acid (NPA) impairs apical hook formation ${ }^{34}$. Hormones such as ethylene and gibberellins (GAs) can skew auxin synthesis and polar transport to one side of plant organs and inhibit cell growth while increasing the same on the contralateral 
(a)

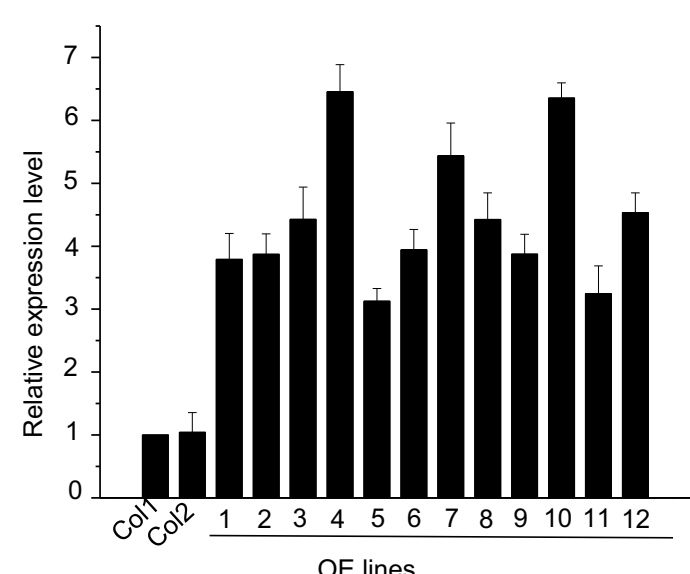

(c)

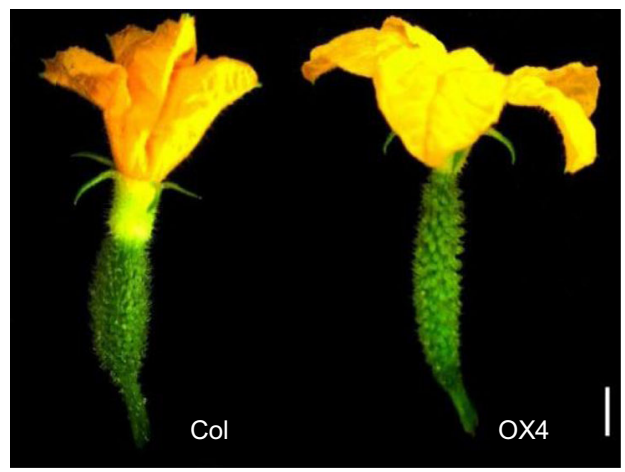

(b)

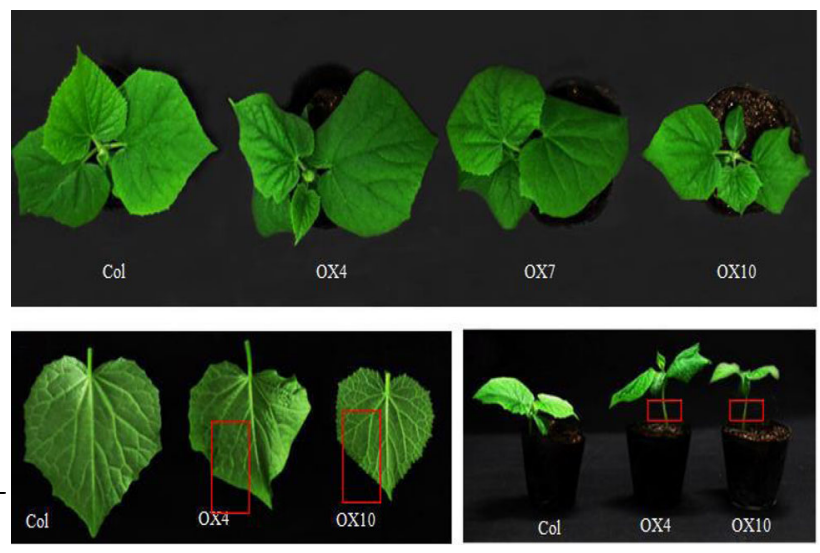

(d)

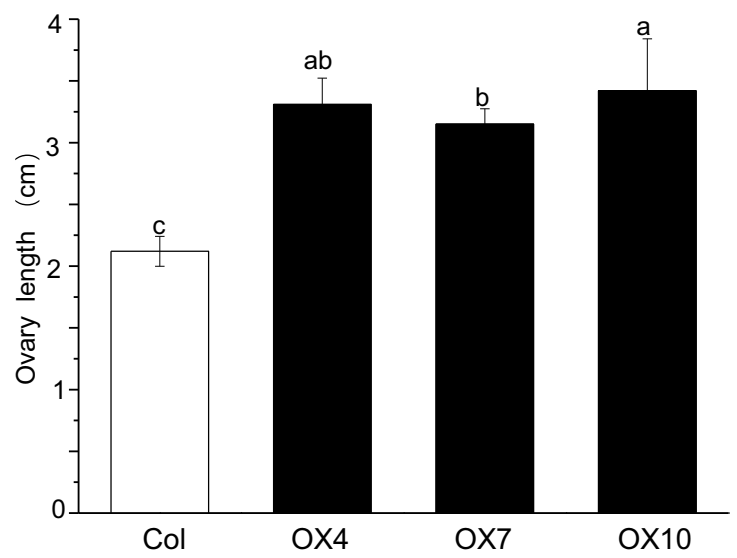

Fig. 5 Auxin hyperaccumulation in CsYUC10b transgenic plants. a CsYUC10b levels in the leaves of control (Col) and CsYUC10b-Overexpressing (OX) plants. b Downward curled leaves and stem elongation of Col and OX. c Ovary growth in Col and OX plants. d Ovary length in Col and OX plants. The data are the means $\left( \pm\right.$ SEs) of three independent experiments, with five replicates each. ${ }^{*} P<0.05$, and ${ }^{* *} P<0.01$

side $^{36}$. In this study, we observed temporal changes in auxin levels during cucumber fruit development. In addition, the auxin concentration was slightly higher on the convex side than on the concave side of the curved fruits at 0-2 DPA, and the differential expression peaked at 4-7 DPA, corresponding to increased curvature. As the curvature decreased from 8 to 18 DPA, the auxin levels gradually became even between both sides. Thus, the angle of fruit curvature is a consequence of differential growth controlled by auxin distribution.

The key step in auxin synthesis is YUCCA-mediated conversion of IPA to IAA ${ }^{37}$. Overexpression of $Y U C$ genes increases auxin production in A. thaliana, while the loss of function of a single $Y U C$ gene does not affect plant growth $^{38}$. However, a previous study showed that mutation at a single $Y U C$ locus prevented the growth of $A$. thaliana and maize seedlings ${ }^{39}$. Typically, YUC-overexpressing lines show phenotypes associated with auxin overproduction. For instance, heterologous expression of
AtYUC6 in potato (Solanum tuberosum) plants increased the auxin concentration, resulting in plants with relatively narrow and downward-curling leaves, relatively long petioles, and increased height and longevity ${ }^{40}$. Similarly, AtYUC6 overexpression in poplar led to rapid shoot growth and slowed the development of the main root, and root hair length increased ${ }^{41}$. In this study, overexpression of $C s Y U C 10 b$ in cucumber resulted in downward-curling leaves and elongated stems and ovaries, which supports the involvement of $C s Y U C 10 b$ in auxin biosynthesis.

$Y U C$ genes also regulate auxin biosynthesis during fruit development. The upregulation of PpYUC11 in peach (Prunus persica) is correlated with increased auxin synthesis and fruit maturation ${ }^{42}$. Likewise, CmYUC6 and CmYUC11 are significantly upregulated in the seeds and fruit mesocarp of melon (Cucumis melo) ${ }^{43}$, and high YUCCA levels in strawberry seeds correlate with auxin biosynthesis and fruit development ${ }^{44}$. CsYUC11 is expressed in the male flowers of A. thaliana, and its 
(a)
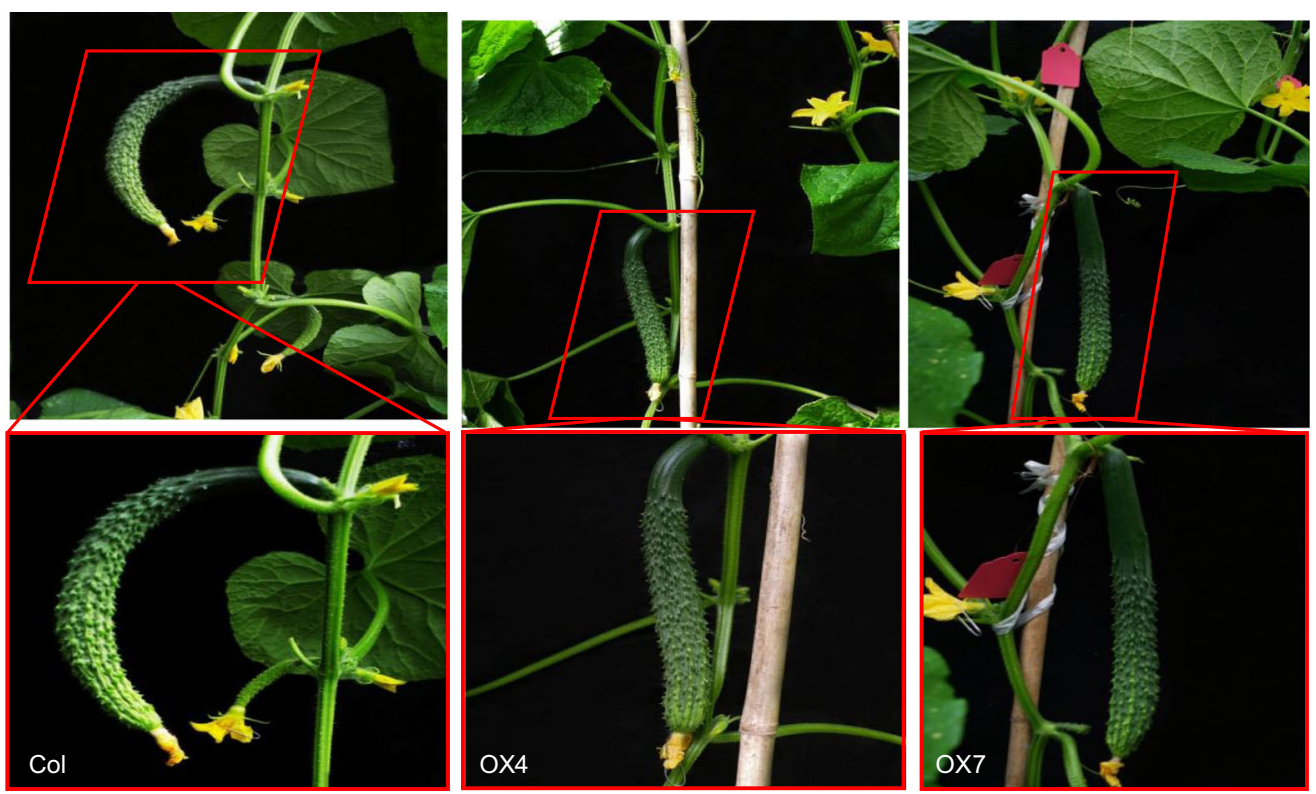

(b)
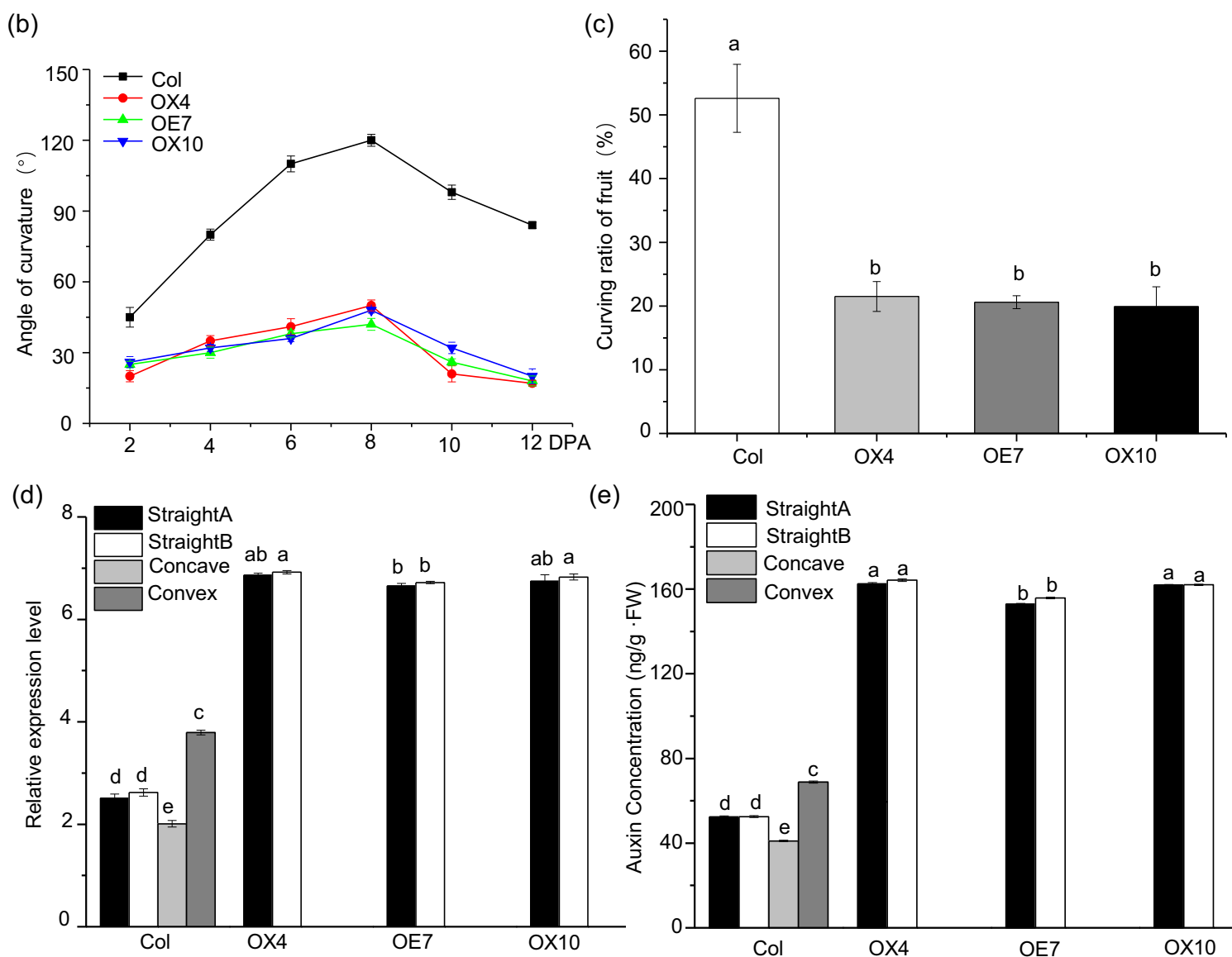

(e) 2007 StraightA

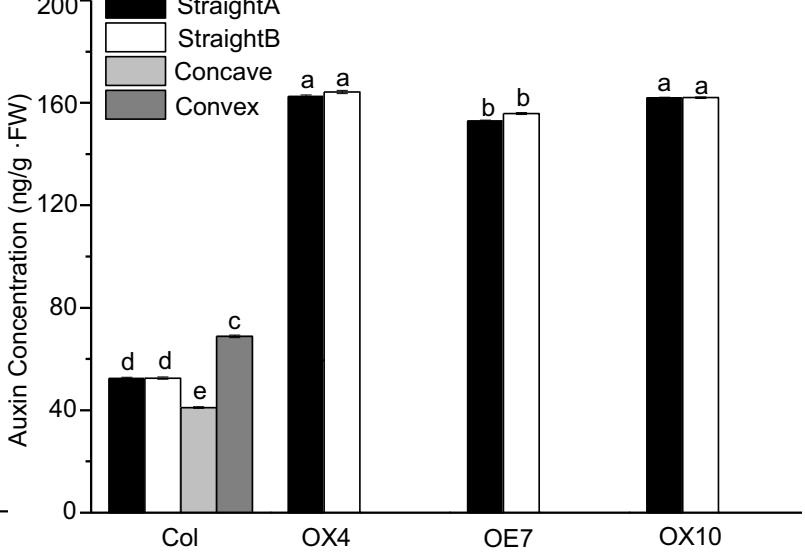

Fig. 6 Effects of CsYUC10b overexpression in cucumber. a Phenotypes of fruits of the control (Col) and CsYUC10b-overexpressing (OX) plants at 4 DPA. $\mathbf{b}$ Angle of fruit curvature in Col and OX plants at 2, 4, 6, 8, and 10 DPA. c Percentage of curved fruits of Col and OX plants. $\mathbf{d}$ CsYUC10b levels on both sides of Col and OX fruits. e Auxin concentration in Col and OX plants at 4 DPA. The data are the means ( \pm SEs) of three independent experiments, with five replicates each $(P<0.05)$ 
overexpression promotes auxin accumulation and elongation of the pedicel and stamen ${ }^{45}$. Consistent with this, overexpression of CsYUC1Ob in cucumber induced symmetric auxin biosynthesis on both sides of the fruits, resulting in the formation of straight fruits.

Fruit development typically progresses from preanthesis ovary growth to ripening ${ }^{46}$. In cucumber, cell division at the earliest stages (0-4 DPA) plays a decisive factor in the final fruit size and shape, and fruit elongation peaks at 4-12 DPA. This is consistent with the findings that rapid cell enlargement occurs after the frequency of cell division decreases ${ }^{47}$. Furthermore, the number of cells in the ovary is also a major factor determining cell division and fruit development ${ }^{48}$. Increased cell numbers and growth in the longitudinal direction determine ovary length and fruit size in cucumber, respectively ${ }^{49}$. In a previous study, we detected shorter cells on the concave side compared to the convex side of curved cucumber fruit at 6 DPA (Supplementary Fig. S8), indicating that fruit curving depends on the tightly regulated differential growth of cells ${ }^{20}$. Since auxin controls both cell division and cell elongation ${ }^{50}$, auxin-dependent differential growth likely progresses through the different developmental phases as follows: (1) establishment of asymmetric auxin biosynthesis that skews cell growth rates, (2) regulation of the increasing-to- maximum phase transition and the spatial stabilization of auxin distribution, (3) control of the maximum-to-decreasing phase transition, and (4) gradual loss of auxin maxima.

Our previous work involved a quantitative trait locus (QTL) analysis of an $F_{2}$ population derived from a cross between the curved-type inbred line L18 and straight-type inbred line D9320. There were 116 pairs of SSR markers applied to these lines; however, only four markers were detected on a linkage group, and the QTL map distance was $2.5 \mathrm{cM}$ on chromosome (chr) $6^{51}$. Given the advancement of genome sequencing technology, RNA sequencing (RNA-seq) has emerged as a comprehensive and accurate tool for analyzing key genes and molecular mechanisms. In this study, we used RNA-seq to identify auxin involvement in fruit curving and screened a candidate gene, CsYUC1Ob (Csa3M190380.1), located on chr 3. Through exogenous auxin (NAA, NOA, and AVG) treatment and gene functional verification, we found that overexpression of CsYUC1Ob promotes the development of straight fruit in cucumber. These results do not account for CsYUC10b, which had no relationship with major effector genes responsible for fruit curving. In our future studies, we propose to utilize the genomic sequences, develop additional markers for QTL fine mapping and identify major genes responsible for fruit curving. This would help us explore the relationship between auxin-coding genes and the other major signaling sequences.

\section{Acknowledgements}

This study was funded by the National Key Research and Development Program for Breeding Seven Key Crops (2016YFD0101700), the Academic Backbone Project of Northeast Agricultural University (18XG06), the Heilongjiang Postdoctoral Scientific Research Developmental Fund (LBHQ16021), and the Natural Science Foundation of Heilongjiang Province, China (LH2019C033). The authors thank Professor Huazhong Ren (College of Agronomy and Biotechnology, China Agricultural University, Beijing) who assisted with the genetic transformation of cucumber.

\section{Author contributions}

S.L., C.W., and Z.Q. designed and conceived the research; S.L., C.W., M.X., and X. Z. performed the experiments; D.L., C.L., and J.L. analyzed the sequencing data; S.L. and C.W. wrote the entire manuscript; and Z.Q. and M.X. edited the manuscript.

\section{Conflict of interest}

The authors declare that they have no conflict of interest.

Supplementary Information accompanies this paper at (https://doi.org/ 10.1038/s41438-020-00354-5).

Received: 16 December 2019 Revised: 11 June 2020 Accepted: 13 June 2020

Published online: 01 September 2020

\section{References}

1. Zhou, X., Qin, Z. W. \& Wang, X. G. Commercial evaluation of cucumber resources. J. Northeast Agric. Univ. 36, 309-316 (2005).

2. Colle, M., Weng, Y., Kang, Y., Ophir, R. \& Grumet, R. Variation in cucumber (Cucumis sativus L.) fruit size and shape results from multiple components acting pre-anthesis and post-pollination. Planta 246, 641-658 (2017).

3. Zang, P., Qin, Z. W., Wang, L. L. \& Zhou, X. Y. Genetic analysis on bent characters of cucumber fruit. J. Northeast Agric. Univ. 18, 1-5 (2011).

4. Grumet, R. \& Colle, M. Genomic analysis of cucurbit fruit growth. Genet. Genomics Cucurbitaceae 20, 321-344 (2016).

5. Pan, Y. et al. Genetic architecture of fruit size and shape variation in cucurbits: a comparative perspective. Theor. Appl. Genet. 133, 1-21 (2019).

6. Xin, T. et al. Genetic regulation of ethylene dosage for cucumber fruit elongation. Plant Cell 31, 1063-1076 (2019).

7. De Jong, M. et al. Solanum lycopersicum auxin response factor 9 regulates cell division activity during early tomato fruit development. J. Exp. Bot. 66, 3405-3416 (2015).

8. De Jong, M., Wolters-Arts, M., Feron, R., Mariani, C. \& Vriezen, W. H. The Solanum lycopersicum auxin response factor 7 (SIARF7) regulates auxin signaling during tomato fruit set and development. Plant J. 57, 160-170 (2009).

9. Ruiz Rosquete, M., Barbez, E. \& Kleine-Vehn, J. Cellular auxin homeostasis: gatekeeping is housekeeping. Mol. Plant. 5, 772-786 (2012).

10. Mashiguchi, K. et al. The main auxin biosynthesis pathway in Arabidopsis. Proc. Natl Acad. Sci. USA 108, 18512-18517 (2011).

11. Stepanova, A. et al. A link between ethylene and auxin uncovered by the characterization of two root-specific ethylene-insensitive mutants in Arabidopsis. Plant Cell 17, 2230-2242 (2005).

12. Benjamin, P. éret et al. Aux/Lax genes encode a family of auxin influx transporters that perform distinct functions during Arabidopsis development. Plant Cell 24, 2874-2885 (2012).

13. Robert, H. S. et al. Plant embryogenesis requires aux/lax-mediated auxin influx. Development 142, 702-711 (2015).

14. Wang, Y. C. \& Guo, H. W. On hormonal regulation of the dynamic apical hook development. N. Phytologist 222, 1230-1234 (2019).

15. Abbas, M., Alabadí, D. \& Blázquez, M. A. Differential growth at the apical hook all roads lead to auxin. Front. Plant Sci. 4, 441 (2013).

16. Tepanova, A. N. et al. The Arabidopsis YUCCA1 flavin monooxygenase functions in the indole-3-pyruvic acid branch of auxin biosynthesis. Plant Cell 23, 3961-3973 (2011).

17. Vandenbussche, F. et al. The auxin influx carriers AUX1 and LAXX are involved in auxin-ethylene interactions during apical hook development in Arabidopsis thaliana seedlings. Development 137, 597-606 (2010). 
18. Liang, X. L. et al. Involvement of cop1 in ethylene-and light-regulated hypocotyl elongation. Planta 236, 1791-1802 (2012).

19. Wang, C. et al. Transcriptome analysis of development pattern of curving fruit in Cucumber. Acta Horticulturae Sin. 41, 2713 (2014).

20. Changjun, G., Zhiwei, Q. \& Xiuyan, Z. Analysis on evaluation method for cucumber fruit bending and relativities. China Vegetables 8, 28-31 (2009).

21. Wang, $\mathbf{C}$. et al. The novel ethylene-responsive factor CsERF025 affects the development of fruit bending in cucumber. Plant Mol. Biol. 95, 519-531 (2017).

22. Patel, R. K. \& Jain, M. NGS QC Toolkit: a toolkit for quality control of next generation sequencing data. PLOS ONE 7, e30619 (2012).

23. Huang, D. W., Sherman, B. T. \& Lempicki, R. A. Systematic and integrative analysis of large gene lists using David bioinformatics resources. Nat. Protoc. 4, 44-57 (2009).

24. Xie, C. et al. KOBAS 2.0: a web server for annotation and identification of enriched pathways and diseases. Nucleic Acids Res. 39, W316-W322 (2011)

25. Wan, $\mathrm{H}$. et al. Selection of appropriate reference genes for gene expression studies by quantitative real-time polymerase chain reaction in cucumber. Anal. Biochem. 399, 257-261 (2010).

26. Schmittgen, T. D. \& Livak, K. J. Analyzing real-time PCR data by the comparative ct method. Nat. Protoc. 3, 1101-1108 (2008).

27. Meng, J. J., Qin, Z. W., Zhou, X. Y. \& Xin, M. An ATP-binding cassette transporter gene from Cucumis sativus L.CSABCA19, is involved in propamocarb stress in Arabidopsis thaliana. Plant Mol. Biol. Rep. 34, 947-960 (2016).

28. Yoo, S., Cho, Y. \& Sheen, J. Arabidopsis mesophyll protoplasts: a versatile cell system for transient gene expression analysis. Nat. Protoc. 2, 1565-1572 (2007).

29. Chen, S., Songkumarn, P., Liu, J. \& Wan, G. A versatile zero background T-vector system for gene cloning and functional genomics. Plant Physiol. 150, 1111-1121 (2009).

30. Jyothishwaran, G. et al. A modified freeze-thaw method for efficient transformation of Agrobacterium Tumefaciens. Curr. Sci. 93, 770-772 (2007).

31. Wang, H., Sui, X., Guo, J., Wang, Z. \& Zhang, Z. Antisense suppression of cucumber (Cucumis sativus L.) sucrose synthase 3 (CSSUS3) reduces hypoxic stress tolerance. Plant Cell Environ. 37, 795-810 (2013).

32. Wang, Y. et al. A GAMYB homologue CSGAMYB1 regulates sex expression of cucumber via an ethylene-independent pathway. J. Exp. Bot. 65, 3201-3213 (2014).

33. Weiler, E., Jourdan, P. \& Conrad, W. Levels of indole-3-acetic acid in intact and decapitated coleoptiles as determined by a specific and highly sensitive solidphase enzyme immunoassay. Planta 153, 561-571 (1981).

34. Zádníková, P. et al. Role of PIN-mediated auxin efflux in apical hook development of Arabidopsis thaliana. Development 137, 607-617 (2010).

35. Raz, V. \& Ecker, J. R. Regulation of differential growth in the apical hook of Arabidopsis. Development 126, 661-3668 (1999).
36. Wang, Y. \& Guo, H. On hormonal regulation of the dynamic apical hook development. N. Phytologist. 222, 1230-1234 (2019).

37. Zhao, Y. Auxin biosynthesis. Arabidopsis Book 12, e0173 (2014).

38. Zhao, Y. et al. A role for flavin monooxygenase-like enzymes in auxin biosynthesis. Science 291, 306-309 (2001).

39. Hou, $X$. et al. Allelic analyses of the Arabidopsis YUC1 locus reveal residues and domains essential for the functions of YUC family of flavin monooxygenases. $J$. Int. Plant Biol. 53, 154-162 (2011).

40. Kim, J. et al. Overexpression of Arabidopsis YUCCA6 in potato results in highauxin developmental phenotypes and enhanced resistance to water deficit Mol. Plant 6, 337-349 (2013).

41. Ke, Q. et al. Transgenic poplar expressing Arabidopsis YUCCA6 exhibits auxinoverproduction phenotypes and increased tolerance to abiotic stress. Plant Physiol. Bioch. 94, 19-27 (2015).

42. Pan, L. et al. PpYUC11, a strong candidate gene for the stony hard phenotype in peach (Prunus persica L. Batsch), participates in IAA biosynthesis during fruit ripening. J. Exp. Bot. 66, 7301-7044 (2015)

43. Zheng, $L$. et al. YUCCA type auxin biosynthesis genes encoding flavin monooxygenasesin melon: Genome-wide identification and developmental expression analysis. S. Afr. J. Bot. 102, 142-152 (2015).

44. Kang, $C$. et al. Genome-scale transcriptomic insights into early-stage fruit development in woodland strawberny Fragaria vesca. Plant Cell 25, 1960-1978 (2013).

45. Yan, S. et al. Different cucumber CSYUC genes regulate response to abiotic stresses and flower development. Sci. Rep. 6, 20760 (2016)

46. Che, G. \& Zhang, X. L. Molecular basis of cucumber fruit domestication. Curr. Opin. Plant Biol. 47, 38-46 (2019).

47. Boonkorkaew, P., Hikosaka, S. \& Sugiyama, N. Effect of pollination on cell division, cell enlargement, and endogenous hormones in fruit development in a gynoecious cucumber. Sci. Hortic. 116, 7 (2008).

48. Jürgen, B. \& Bangerth, F. Cell number, cell size and hormone levels in semiisogenic mutants of lycopersicon pimpinellifolium differing in fruit size. Physiol. Plant. 72, 316-320 (1988).

49. Yang, X. Y. et al. Characterization and expression profiling of cucumber kinesin genes during early fruit development: revealing the roles of kinesins in exponential cell production and enlargement in cucumber fruit. J. Exp. Bot. 64, 4541-4557 (2013)

50. Chen, J. G. Dual auxin signaling pathways control cell elongation and division. J. Plant Growth Regul. 20, 255-264 (2001).

51. Zhang, P. et al. Mapping quantitative traits loci and proteomics studies on bending of cucumber fruit. Journal of Northeast Agric. Univ. 41, 28-21 (2009). 